\title{
Exploración del uso de modelos celulares con la estrategia de clase invertida
}

Exploration of the use of cellular models with the inverted class strategy

\section{RESUMEN}

En el contexto de aprendizaje significativo se realizó exploración y diseño de una estrategia didáctica basada en el Desarrollo de modelos de biología celular y molecular para problemas relacionados con el habla, audición y el lenguaje, con una estrategia de aula invertida en estudiantes de fonoaudiología - ciencias de la salud de la Universidad del Cauca. Se muestran algunos ejemplos de resultado de la aplicación de esta estrategia.

PALABRAS CLAVE: Modelos, Biología Celular, Clase invertida.

\section{ABSTRACT}

In the context of learning, work, reading, design, strategy, communication, development, models, cellular and molecular biology, problems related to speech, hearing and language, the strategy of investment in phonoaudiology students. of the health of the University of Cauca. Some examples of results of the application of this strategy are shown.

KEYWORDS: Newton's Laws, mobile app, Higher Education, gamification.

\footnotetext{
${ }^{1}$ Biólogo Magíster en Bioquimica.1. Docente. Departamento de Ciencias Fisiológicas. Facultad de Ciencias de la Salud. Universidad del Cauca. Colombia. andresquiroga@ unicauca.edu.co.

${ }^{2}$ Química. Magister Ciencias Bioquímica 2. Departamento de Ciencias Fisiológicas. Docente. Facultad de Ciencias de la Salud. Universidad del Cauca. Colombia. sifreyre@unicauca.edu.co.
} 


\section{Descripción del Problema}

Uno de los componentes de las pruebas obligatorias de estado para profesionales o pruebas Saber Pro es la medición de capacidades de estudiantes de Ciencias de la Salud en Competencias como la resolución de problemas, por lo que se hace necesario implementar metodologías de clase que relacionen aspectos y promuevan en los estudiantes la adquisición de aprendizajes para resolver situaciones en contexto desde las áreas básicas como por ejemplo desde la asignatura biología celular y molecular.

\section{Pregunta de investigación}

¿Cómo desarrollar aprendizaje significativo y capacidad de resolución de problemas a través del uso de aula invertida en estudiantes de primer semestre de fonoaudiología?

\section{Objetivos}

Implementar la estrategia de aula invertida mediante el uso de modelos celulares y solución de problemas en estudiantes de primer semestre de fonoaudiología.

Fortalecer la comprensión de los conceptos de biología celular y molecular a través de las herramientas de aprendizaje asociativo y colaborativo.

ñol.

\section{Metodología}

El aprendizaje significativo presenta varias dimensiones entre las cuales motivación, comprensión, práctica y la participación activa tanto de estudiantes y docentes son pieza clave para lograr un resultado de enseñanza aprendizaje superior. Comparando los métodos tradicionales con los utilizados para el fortalecimiento de aprendizaje activo, se representa como un reto que debe ser planeado paso a paso por el docente orientador, estandarizarlo, crear estrategias de seguimiento y hacer una evaluación adecuada que permita realizar una correcta implementación del método en los grupos de trabajo (3). Los estudiantes deberán adquirir saberes, comprender e interpretar estos dentro de un contexto y organizarlos para su aplicación (4) de esta forma dar solución al interrogante de fonoaudiología mediante la elaboración de un modelo celular, lo cual sin lugar a dudas permite fortalecer competencias del saber, del hacer y por ultimo competencias blandas (transversales); siendo las ultimas aquellas que le permiten mejorar el trabajo en equipo para resolver una situación problémica a partir de conceptos previos propios de su nivel de aprendizaje.

El abordaje metodológico en clase comprendió cuatro momentos: en primer lugar el docente determina los temas e interrogantes a trabajar durante el desarrollo del problema y los tiempos necesarios para lograrlo, de igual forma se organizan los grupos de trabajo de estudiantes a los cuales se les indica una situación problema en contexto con la asignatura biología celular y el aspecto fonoaudiológico a desarrollar. Un segundo momento es cuando los grupos de estudiantes discuten las posibles soluciones al interrogante fonoaudiológico en consonancia a la revisión de tema previa y a las orientaciones del docente tutor, en este mismo momento los estudiantes deciden el diseño o estilo de modelo celular más adecuado para dar respuesta al problema planteado. En éste momento es clave, la orientación por parte del docente para que el grupo de estudiantes pueda delimitar con claridad la o las respuestas a su interrogante fonoaudiológico. El tercer momento, corresponde a la fase de modelación celular, en la cual el grupo de estudiantes hace evidente su solución del interrogante fonoaudiológico a través del diseño de maquetas de las estructuras celulares o moleculares implicadas en el problema planteado. En esta fase del proceso, los estudiantes evidencian sus niveles de creatividad y el grado de conocimiento que poseen acerca del interrogante planteado. El cuarto momento corresponde a la socialización de la respuesta al interrogante para lo cual se evaluó mediante una rúbrica de evaluación preestablecida y diseñada por el docente y que los estudiantes conocieron previo al inicio de la actividad. Lo anteriormente descrito se halla consignado en la figura 1 . 
- Se plantea el problema a resolver en el ámbito de la fonoaudiología.

- Estudiantes discuten en grupo, buscando alternativas de solución.

- evaluación
mediante el uso de
rubricas con
criterios
establecidos al
inicio del
desarrollo de la
actividad.

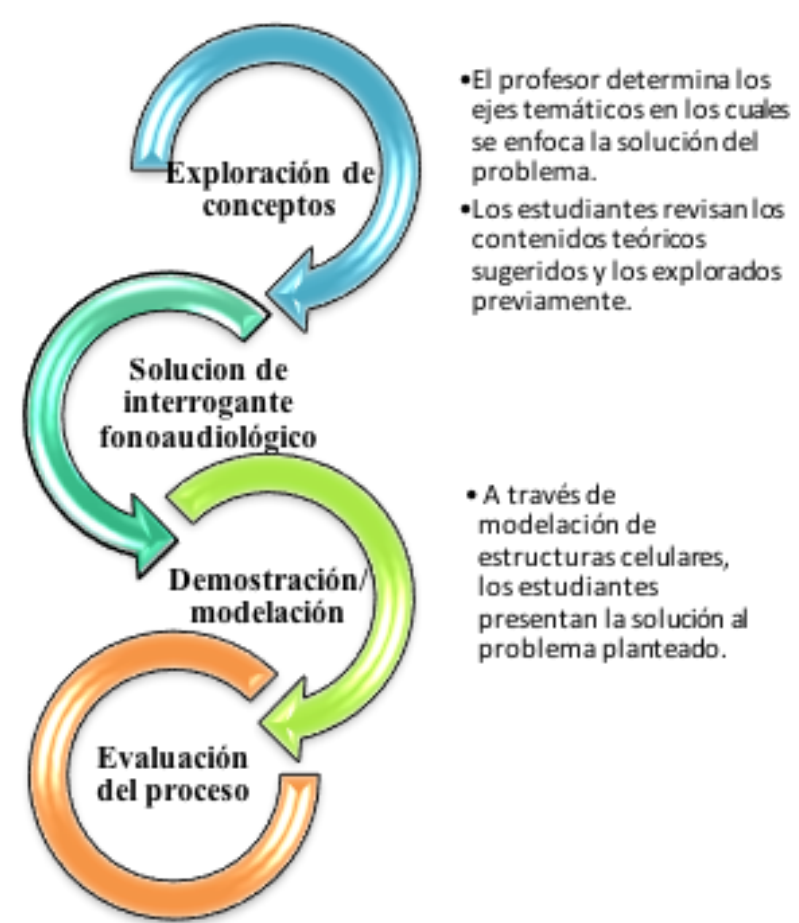

Figura 1.Desarrollo metodológico que muestra los procesos metodológicos de la estrategia pedagógica utilizada (indagación, resolución, modelación y evaluación) para la resolución problema fonoaudiológico mediante un modelación en el contexto de biología celular en estudiantes de primer semestre Fonoaudiología, Facultad de ciencias de la salud 2018.

Se pudo evaluar diferentes aspectos de biología celular y molecular, algunos de las temáticas que más abordaron los estudiantes fueron: desarrollo cognitivo, funcionamiento de los organelos celulares y características del oído humano, con la estructura y funcionamiento celular en la audición (Tabla 1). En este último aspecto se realizaron modelos correspondientes a órganos especializados de la función auditiva, como puede verse en la tabla 1.

Basándonos en los aspectos se pudo realizar la implementación de la metodología, para la resolución de aspectos fonoaudiológicos en relación a contexto de temas básicos en biología celular y molecular.

La estrategia de aula invertida es un método que entre otras características considera como elemento central, la selección de las competencias que el estudiante deba desarrollar, en esto, el docente realiza la clasificación de las temáticas y problemas que los estudiantes desarrollan, explican y modelan basados en sus aprendizajes de la asignatura.

Tabla 1. Resultados de estrategia de resolución de problemas basado en aula invertida

\begin{tabular}{|c|c|c|}
\hline Interrogante Fonoaudiológico & Temática Curso & Modelo desarrollado \\
\hline $\begin{array}{l}\text { 1. Paciente que repite la misma palabra pero } \\
\text { no comprende la mayoría de las cosas que } \\
\text { se le dicen. Puede articular sin dificultad } \\
\text { pero la escritura y la lectura también } \\
\text { suelen ser defectuosas, con numerosos } \\
\text { errores en la ortografía y de omisión de } \\
\text { letras. } \\
\text { 2. Paciente que se observa con la principal } \\
\text { dificultad cuando habla, ya que utiliza muy } \\
\text { pocas palabras y no puede articularlas de } \\
\text { forma coherente. Es decir, su habla no es } \\
\text { fluida y sus oraciones a menudo no son } \\
\text { gramaticalmente correctas. }\end{array}$ & $\begin{array}{l}\text { Estructura y función de orgánulos } \\
\text { celulares. }\end{array}$ & $\begin{array}{l}\text { Áreas de desarrollo de } \\
\text { lenguaje, área de Broca y } \\
\text { Wernicke }\end{array}$ \\
\hline
\end{tabular}




\begin{tabular}{|l|l|l|l|}
\hline 3. $\begin{array}{l}\text { Auditivo. Cuáles son las células encargadas } \\
\text { de diferenciar un sonido agudo de uno } \\
\text { grave. }\end{array}$ & $\begin{array}{l}\text { Cito esqueleto, células ciliares } \\
\text { auditivas. }\end{array}$ & $\begin{array}{l}\text { Modelo de órgano de corti } \\
\text { en el oído humano. }\end{array}$ \\
\hline $\begin{array}{l}\text { 4. Paciente que presenta irritación y malestar } \\
\text { en zona auditiva, no puede escuchar } \\
\text { adecuadamente. }\end{array}$ & $\begin{array}{l}\text { Proceso inflamatorio diferencia } \\
\text { entre apoptosis y necrosis }\end{array}$ & $\begin{array}{l}\text { Modelo de Coclea en el oído } \\
\text { humano. }\end{array}$ \\
\hline $\begin{array}{l}\text { Paciente con malestar fiebre e } \\
\text { inflamación de faringe, asociada con } \\
\text { pérdida de control y vértigo. }\end{array}$ & $\begin{array}{l}\text { Ciclo celular y Diferenciación } \\
\text { Celular }\end{array}$ & Modelo de faringe \\
\hline
\end{tabular}

Como se mencionó en la metodología, en el momento tres de proceso demostración / modelación (Figura 1); el grupo de trabajo de estudiantes realiza el diseño celular con el fin de responder al interrogante fonoaudiológico formulado. En la figura 2 se presentan algunos

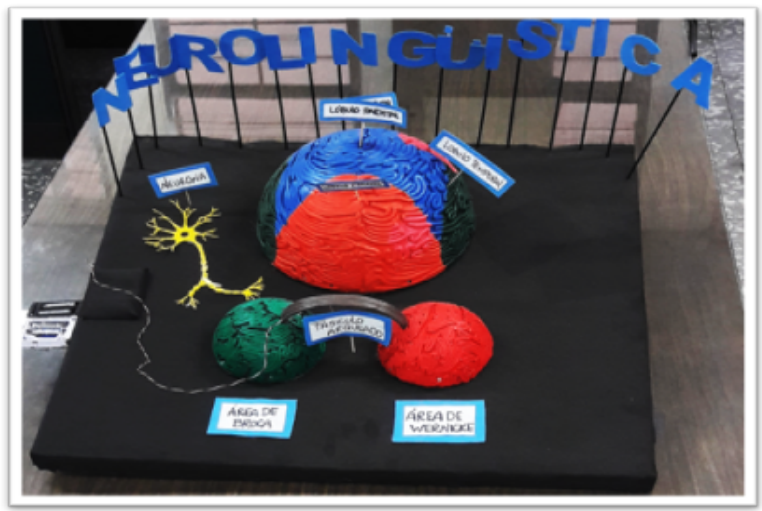

Áreas de desarrollo de lenguaje

Figura 2. Modelos de biología celular

Los modelos celulares que se muestran en la figura 2 corresponden al interrogante fonoaudiológico 1 y 2 (representado en la maqueta $2 \mathrm{a}$ ) y al interrogante 3 (representado en la maqueta $2 \mathrm{~b}$ ) que se resumen en la tabla 1 antes descrita.

Con el desarrollo de esta actividad se pudo mejorar en los estudiantes el desarrollo de varias competencias y habilidades como en manejo de tiempo, socialización y apertura que hizo en los estudiantes demostraran capacidad de trabajo, creatividad e iniciativa, que claramente se ve reflejada en los modelos mostrados previamente en la figura 2. Basándonos en su desarrollo podríamos sugerir esta herramienta como una forma factible de incorporar en la práctica docente de ciencias básicas, como bien lo pudo expresar Nalon

"Saia da posição de "invejoso" e vá para posição ejemplos de los modelos realizados por los estudiantes de fonoaudiología de primer semestre de la asignatura biología celular y molecular.

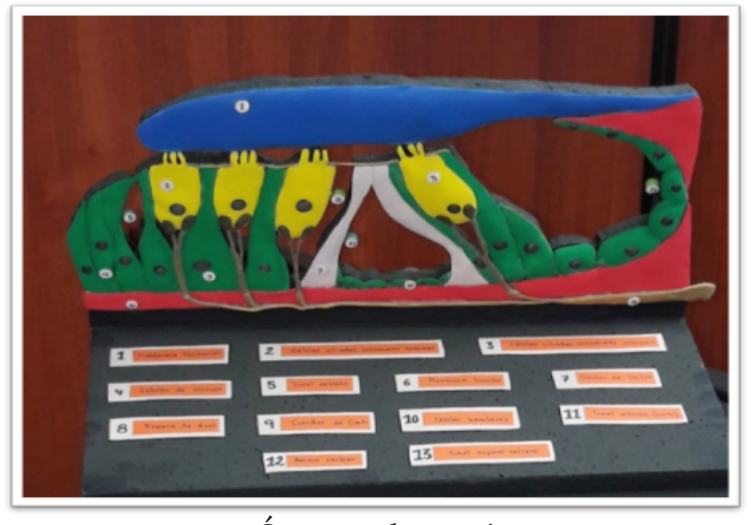

Órgano de corti

de "aprendiz" com a modelagem" (5) se podría salir la posición de envidia y pasar a la posición de aprendiz con el modelado.

\section{Conclusión}

En un contexto, el aprendizaje significativo puede permitir la exploración y diseño de modelos de biología celular y molecular para problemas relacionados con el habla, audición y el lenguaje usando la estrategia de aula invertida en estudiantes de fonoaudiología -ciencias de la salud de la Universidad del Cauca.

El cambio de metodología exige un gran esfuerzo del docente en la planeación, ejecución y evaluación de resultados, al igual que el tiempo invertido por los estudiantes es superior a la ejecución de 
una clase tradicional y exige trabajo individual y en equipo. Se recomienda la revisión de material y el continuo diseño de nuevas actividades encaminadas a fortalecer la capacidad de resolución de problemas, de esta forma se le agrega mayor dinamismo al proceso de enseñanza y aprendizaje de las ciencias básicas en programas académicos de ciencias de la salud, como el programa de fonoaudiología. Además, estos mecanismos participativos refuerzan la consecución de algunas competencias transversales que son importantes que se desarrollen en los estudiantes desde sus inicios profesionales y que en la mayoría de las ocasiones son complicadas de conseguir cuando se dispone de muchos alumnos en el aula.

\section{REFERENCIAS}

[1]. Nwosisi, C., Ferreira, A., Rosenberg, W., \& Walsh, K. (2016). A study of the flipped classroom and its effectiveness in flipping thirty percent of the course content. International Journal of Information and Education Technology, 6(5), 348.

[2]. Martínez Olvera, W., Esquivel Gámez, I., \& Castillo, J. M. (2014). Aula Invertida o Modelo Invertido de Aprendizaje: origen, sustento e implicaciones. Recuperado de https://www. researchgate. net/ publication/273765424_Aula_Invertida_o_ Modelo_Invertido_de_Aprendizaje_origen_sustento_e_implicaciones.

[3]. Carranza Alcántar, María del Rocío. (2017). Enseñanza y aprendizaje significativo en una modalidad mixta: percepciones de docentes y estudiantes. RIDE. Revista Iberoamericana para la Investigación y el Desarrollo Educativo, 8(15), 898-922. https://dx.doi. org/10.23913/ride.v8i15.326

[4]. National Research Council. 2000. How People Learn: Brain, Mind, Experience, and School: Expanded Edition. Washington, DC: The National Academies Press. Pag 52 - 68 https://doi.org/10.17226/9853. https:// www.nap.edu/read/9853/chapter/6\#67

[5] Nalón Carolina. 2015 [ARTIGO] Vai ver tem algo que você precisa aprender. Recuperado de: Posted on setembro 20, 2015 by Carolina Nalon. Recuperoado de: http://carolinanalon.com/artigo-vai-vertem-algo-que-voce-precisa-aprender/ 